\title{
Cooperative Multi-Sensor Detection under Variable-Length Coding
}

\author{
Mustapha Hamad \\ LTCI, Telecom Paris, IP Paris \\ 91120 Palaiseau, France \\ mustapha.hamad@telecom-paris.fr
}

\author{
Michèle Wigger \\ LTCI, Telecom Paris, IP Paris \\ 91120 Palaiseau, France \\ michele.wigger@telecom-paris.fr
}

\author{
Mireille Sarkiss \\ SAMOVAR, Telecom SudParis, IP Paris \\ 91011 Evry, France \\ mireille.sarkiss@telecom-sudparis.eu
}

\begin{abstract}
We investigate the testing-against-independence problem over a cooperative MAC with two sensors and a single detector under an average rate constraint on the sensors-detector links. For this setup, we design a variable-length coding scheme that maximizes the achievable type-II error exponent when the type-I error probability is limited to $\epsilon$. Similarly to the single-link result, we show here that the optimal error exponent depends on $\epsilon$ and that variable-length coding allows to increase the rates over the optimal fixed-length coding scheme by the factor $(1-\epsilon)^{-1}$.

Index Terms-Distributed Hypothesis Testing, Cooperative MAC, Variable-Length Coding, Error Exponent
\end{abstract}

\section{INTRODUCTION}

Motivated by the broadly emerging Internet of Things (IoT) applications, distributed hypothesis testing problems gained increasing attention recently. In such problems, sensors send information about their observations to one or multiple decision centers. Then, the decision centers attempt to detect the joint distributions underlying the data observed at all the terminals including their own observations.

Our focus is on binary hypothesis testing with a null hypothesis and an alternative hypothesis. We are interested in maximizing the exponential decay (in the number of observed samples) of the probability of error under the alternative hypothesis, given a constraint on the probability of error under the null hypothesis. The study of such a Stein setup has a long history in the information theoretic literature, see e.g., [1]-[8] which study point-to-point, interactive, cascaded, and multi-sensor and/or multi-detector systems. All these works constrain the maximum rate of communication between terminals, and a fixed-length communication scheme is obviously optimal. Recently, the authors of [9] proposed to only constrain the average rate of communication, and they presented a variable-length coding scheme that under this weaker constraint improves the maximum achievable error exponent. The present work is the first extension of the point-to-point average-rate scenario in [9] and the corresponding variablelength coding scheme to systems with multiple sensors.

Specifically, we consider the two-sensors single-detector system in Fig. 1, where the first sensor communicates over a shared link to the second sensor and the detector, and after receiving this message, also the second sensor communicates with the detector. The two sensors observe the sequences $X_{1}^{n}$ and $X_{2}^{n}$, respectively, and the detector observes $Y^{n}$, where we assume that the following Markov chain holds both under the null hypothesis $\mathcal{H}=0$ as well as under the alternative hypothesis $\mathcal{H}=1$ :

$$
X_{1}^{n} \leftrightarrow X_{2}^{n} \leftrightarrow Y^{n}
$$

We consider the testing-against-independence scenario where under the alternative hypothesis $\mathcal{H}=1$ the observations at the two sensors are independent of the observations at the detector. We further assume that the sensors' observations $X_{1}^{n}, X_{2}^{n}$ follow the same joint distribution and the decision center's observation $Y^{n}$ follows the same marginal distribution under both hypotheses. A more general version of our problem (without Markov chain (1)) was studied in [10], but under a maximum rate constraint.

In this paper, we characterize the maximum achievable error exponent $\theta_{\epsilon}^{*}\left(R_{1}, R_{2}\right)$ under the alternative hypothesis when the error probability under the null hypothesis is not allowed to exceed $\epsilon$, and where here $R_{1}$ and $R_{2}$ denote the rates of communication from the first and the second sensors, respectively. As we show in this paper, and in contrast to the optimal error exponent under a maximum rate constraint $\theta_{\epsilon \text {,Fix }}^{*}\left(R_{1}, R_{2}\right)[10],{ }^{1}$ the optimal exponent $\theta_{\epsilon}^{*}\left(R_{1}, R_{2}\right)$ depends on $\epsilon$. In fact, as a main result, we obtain

$$
\theta_{\epsilon}^{*}\left(R_{1}, R_{2}\right)=\theta_{\epsilon, \mathrm{Fix}}^{*}\left(R_{1} /(1-\epsilon), R_{2} /(1-\epsilon)\right) .
$$

Thus, through variable-length coding we can increase all available rates in the network by the factor $(1-\epsilon)^{-1}$. A similar observation was already made for the point-to-point setup studied in [9]. In this sense, the current paper extends the conclusion to multiple links, and it shows in particular that the rate-increase can be attained on all links simultaneously.

Notation: We follow the notation in [11] and [9]. In particular, we use sans serif font for bit-strings: e.g., $\mathrm{m}$ for a deterministic and $M$ for a random bit-string. We let string $(m)$ denote the shortest bit-string representation of a positive integer $m$, and for any bit-string $\mathrm{m}$ we let len $(\mathrm{m})$ and $\operatorname{dec}(\mathrm{m})$ denote its length and its corresponding positive integer. We use $h_{\mathrm{b}}(\cdot)$ for the binary entropy function.

\footnotetext{
${ }^{1}$ In the converse proof of [10, Theorem 2], the second step used to upper bound the rate $R_{1}$ relies on the Markov chain $X_{1 i} \leftrightarrow\left(M_{1}, X_{1}^{i-1} X_{2}^{i-1}\right) \leftrightarrow$ $X_{2_{i+1}}^{n}$, which does not necessarily hold. The result of [10] remains however valid under the Markov chain (1), see Remark 2 ahead.
} 


\section{SySTEM MODEL}

Consider the distributed hypothesis testing problem in Fig. 1 in the special case of testing against independence where

$$
\begin{aligned}
& \text { under } \mathcal{H}=0:\left(X_{1}^{n}, X_{2}^{n}, Y^{n}\right) \sim \text { i.i.d. } P_{X_{1} X_{2}} \cdot P_{Y \mid X_{2}} \\
& \text { under } \mathcal{H}=1:\left(X_{1}^{n}, X_{2}^{n}, Y^{n}\right) \sim \text { i.i.d. } P_{X_{1} X_{2}} \cdot P_{Y} .
\end{aligned}
$$

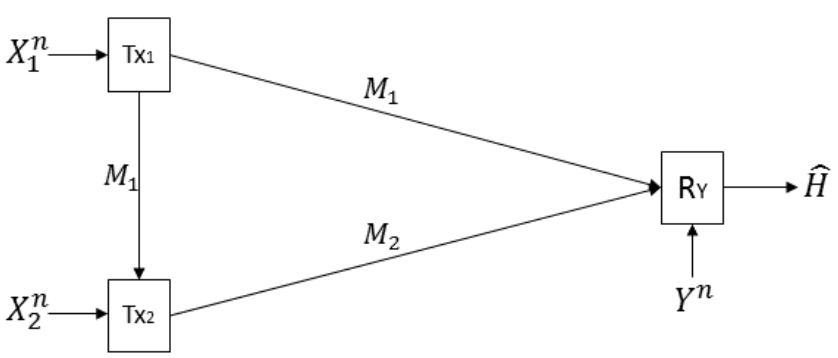

Fig. 1. Cooperative MAC Setup with 2 transmitters and 1 receiver.

Specifically, the system consists of two transmitters $\left(T_{X_{1}}\right.$ and $\left.T_{X_{2}}\right)$ and a receiver $\left(R_{Y}\right) . T_{X_{1}}$ observes the source sequence $X_{1}^{n}$ and sends its bit-string message $\mathrm{M}_{1}=\phi_{1}^{(n)}\left(X_{1}^{n}\right)$ to both $T_{X_{2}}$ and $R_{Y}$, where the encoding function is of the form $\phi_{1}^{(n)}: \mathcal{X}_{1}^{n} \rightarrow\{0,1\}^{\star}$ and satisfies the rate constraint

$$
\mathbb{E}\left[\operatorname{len}\left(\mathrm{M}_{1}\right)\right] \leq n R_{1} \text {. }
$$

$T_{X_{2}}$ observes the source sequence $X_{2}^{n}$ and with the message $M_{1}$ received from $T_{X_{1}}$, it computes the bit-string message $\mathrm{M}_{2}=\phi_{2}^{(n)}\left(X_{2}^{n}, \mathrm{M}_{1}\right)$ using some encoding function $\phi_{2}^{(n)}$ : $\mathcal{X}_{2}^{n} \times\{0,1\}^{\star} \rightarrow\{0,1\}^{\star}$ satisfying the rate constraint

$$
\mathbb{E}\left[\operatorname{len}\left(\mathrm{M}_{2}\right)\right] \leq n R_{2} \text {. }
$$

$T_{X_{2}}$ sends message $\mathrm{M}_{2}$ to $R_{Y}$ which decides on the hypothesis $\mathcal{H}=\{0,1\}$ based on the messages $\mathrm{M}_{1}$ and $\mathrm{M}_{2}$ and its own observation $Y^{n}$. That means, using a decoding function $g^{(n)}$ : $\mathcal{Y}^{n} \times\{0,1\}^{\star} \times\{0,1\}^{\star} \rightarrow\{0,1\}$, it produces:

$$
\hat{\mathcal{H}}=g^{(n)}\left(\mathrm{M}_{1}, \mathrm{M}_{2}, Y^{n}\right) \in\{0,1\} .
$$

The goal is to design encoding and decision functions such that their type-I error probability

$$
\alpha_{n} \triangleq \operatorname{Pr}[\hat{\mathcal{H}}=1 \mid \mathcal{H}=0]
$$

stays below a given threshold and the type-II error probability

$$
\beta_{n} \triangleq \operatorname{Pr}[\hat{\mathcal{H}}=0 \mid \mathcal{H}=1]
$$

decays to 0 exponentially fast.

Definition 1: Error exponent $\theta \geq 0$ is called $\epsilon$-achievable if there exists a sequence of encoding and decision functions $\left\{\phi_{1}^{(n)}, \phi_{2}^{(n)}, g^{(n)}\right\}$ satisfying

$$
\begin{aligned}
\alpha_{n} & \leq \epsilon, \\
\varlimsup_{n \rightarrow \infty} \inf \frac{1}{n} \log \frac{1}{\beta_{n}} & \geq \theta .
\end{aligned}
$$

The supremum over all $\epsilon$-achievable error exponents is called the optimal error exponent and is denoted $\theta_{\epsilon}^{*}\left(R_{1}, R_{2}\right)$.
Remark 1: The present setup differs from the one considered by Zhao and Lai [10] only in that [10] imposes the more stringent constraints

$$
\text { len }\left(\mathrm{M}_{i}\right) \leq n R_{i}, \quad i \in\{1,2\},
$$

instead of the expected rate constraints (5) and (6). Under the rate-constraints (12), without loss of optimality, the two transmitters can send messages $M_{1}$ and $M_{2}$ of fixed lengths.

\section{MAIN RESULTS}

Theorem 1: There exist auxiliary random variables $U_{1}$ and $U_{2}$ such that the optimal error exponent is given by:

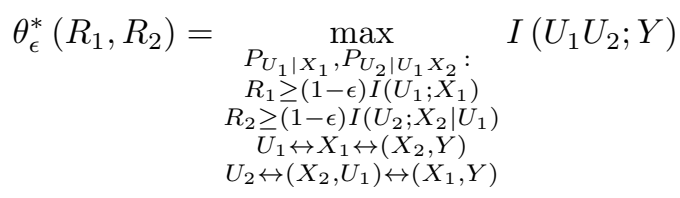

where mutual information quantities are calculated according to the joint pmf $P_{U_{1} U_{2} X_{1} X_{2} Y} \triangleq P_{U_{1} \mid X_{1}} P_{U_{2} \mid U_{1} X_{2}} P_{X_{1} X_{2}} P_{Y \mid X_{2}}$.

Proof: Achievability is proved in Section IV and the converse in Section V.

Lemma 1: In Theorem 1, it suffices to choose $U_{1}$ and $U_{2}$ over alphabets of sizes $\left|\mathcal{U}_{1}\right| \leq\left|\mathcal{X}_{1}\right|+2$ and $\left|\mathcal{U}_{2}\right| \leq\left|\mathcal{U}_{1}\right|\left|\mathcal{X}_{2}\right|+1$.

Proof: Omitted. It follows by standard applications of Carathéodory's theorem, see [11, Appendix C].

\section{A. Comparing Variable-Length with Fixed-Length Coding}

For comparison, we also present the optimal error exponent under fixed-length coding.

Remark 2: Under fixed-length coding, i.e., under rate constraints (12), the optimal error exponent $\theta_{\epsilon \text {,Fix }}^{*}\left(R_{1}, R_{2}\right)$ is [10]:

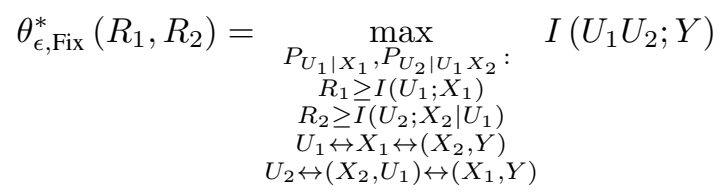

where mutual information quantities are calculated according to the joint pmf $P_{U_{1} U_{2} X_{1} X_{2} Y} \triangleq P_{U_{1} \mid X_{1}} P_{U_{2} \mid U_{1} X_{2}} P_{X_{1} X_{2}} P_{Y \mid X_{2}}$.

Proof: Achievability can be proved as described in Section IV when the set $\mathcal{S}_{n}$ is replaced by an empty set. The converse can be shown as in Section $\mathrm{V}$ if inequality (76), i.e., $H\left(\tilde{\mathrm{M}}_{i}\right) \leq \frac{n R_{i}}{\Delta_{n}}\left(1+h_{b}\left(\frac{\Delta_{n}}{n R_{i}}\right)\right)$, is replaced by the trivial inequality $H\left(\tilde{\mathrm{M}}_{i}\right) \leq n R_{i}$. A more direct proof is also possible, similar to the one in [10]; the converse proof in [10] relies however on a wrong Markov chain, see our footnote 1.

We examine the gain provided by variable-length coding on the cooperative MAC at hand of an example. Let $X_{1}, S, T$ be independent Bernoulli random variables of parameters $a, p, q \in[0,1]$ and set $X_{2}=X_{1} \oplus T$ and $Y=X_{2} \oplus S$. For this example, Fig. 2 plots the optimal error exponents of variable-length and fixed-length coding under a sum-rate constraint:

$$
\begin{aligned}
& \theta_{\epsilon, \Sigma}^{*}(R):=\max _{R_{1}, R_{2} \geq 0: R_{1}+R_{2} \leq R} \theta_{\epsilon}^{*}\left(R_{1}, R_{2}\right) \\
& \theta_{\epsilon, \mathrm{Fix}, \Sigma}^{*}(R):=\max _{R_{1}, R_{2} \geq 0: R_{1}+R_{2} \leq R} \theta_{\epsilon, \mathrm{Fix}}^{*}\left(R_{1}, R_{2}\right)
\end{aligned}
$$


for $\epsilon=0.07$ and in function of the sum-rate $R$.

Note that the optimal type-II error exponent under an expected rate constraint $R$ coincides with the optimal type-II error exponent under a maximum rate constraint $(1-\epsilon) R$. Moreover, as $R$ increases, both error exponents $\theta_{\epsilon, \Sigma}^{*}$ and $\theta_{\epsilon, \text { Fix }, \Sigma}$ tend to the optimal exponent $I\left(X_{1} X_{2} ; Y\right)$ that can be obtained in a central hypothesis testing problem where the detector directly observes all theses sequences $X_{1}^{n}, X_{2}^{n}, Y^{n}$. In particular, both simulated optimal error exponents reach a value of 0.7011 at $R=1.1$ which is almost $98.25 \%$ of $I\left(X_{1} X_{2} ; Y\right)=0.7136$.

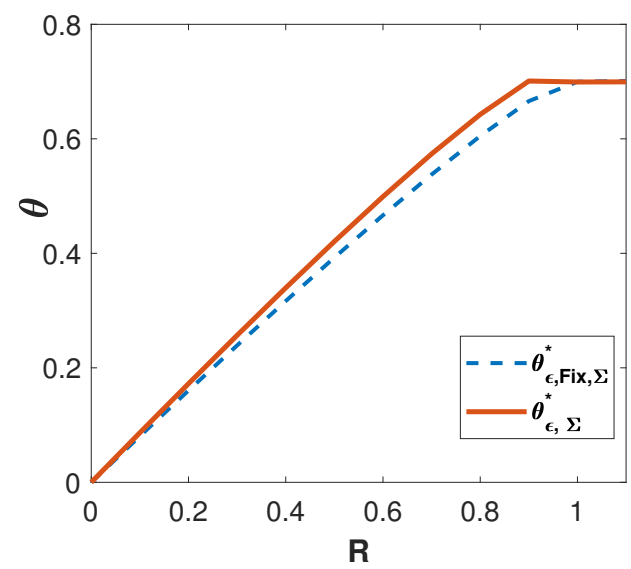

Fig. 2. Optimal exponents under variable-length and fixed-length coding under a sum-rate constraint for above example with $a=0.5, p=0.75, q=$ 0.95 , and $\epsilon=0.07$.

\section{Achievability Proof}

Fix a large blocklength $n$, a small number $\mu \in(0, \epsilon)$, and conditional pmfs $P_{U_{1} \mid X_{1}}$ and $P_{U_{2} \mid U_{1} X_{2}}$ such that:

$$
\begin{gathered}
R_{1}=(1-\epsilon+\mu)\left(I\left(U_{1} ; X_{1}\right)+2 \mu\right) \\
R_{2}=(1-\epsilon+\mu)\left(I\left(U_{2} ; X_{2} \mid U_{1}\right)+2 \mu\right)
\end{gathered}
$$

where mutual information quantities are calculated according to the joint pmf

$$
P_{U_{1} U_{2} X_{1} X_{2} Y} \triangleq P_{U_{1} \mid X_{1}} \cdot P_{U_{2} \mid U_{1} X_{2}} \cdot P_{X_{1} X_{2}} \cdot P_{Y \mid X_{2}} .
$$

Randomly generate a codebook

$$
C_{U_{1}} \triangleq\left\{u_{1}^{n}\left(m_{1}\right): m_{1} \in\left\{1, \cdots, 2^{n\left(I\left(U_{1} ; X_{1}\right)+\mu\right)}\right\}\right\}
$$

by drawing all entries i.i.d. according to the marginal pmf $P_{U_{1}}$. For each codeword $u_{1}^{n}\left(m_{1}\right)$, generate a codebook

$C_{U_{2}}\left(m_{1}\right) \triangleq\left\{u_{2}^{n}\left(m_{2} \mid m_{1}\right): m_{2} \in\left\{1, \cdots, 2^{n\left(I\left(U_{2} ; X_{2} \mid U_{1}\right)+\mu\right)}\right\}\right\}$,

by drawing the $j$-th entry of each codeword according to the marginal pmf $P_{U_{2} \mid U_{1}}$. Also, choose a subset $\mathcal{S}_{n}$ of the typical set $\mathcal{T}_{\mu}^{(n)}\left(P_{X_{1}}\right)$ with probability slightly less than $\epsilon$ :

$$
\mathcal{S}_{n} \subseteq \mathcal{T}_{\mu}^{(n)}\left(P_{X_{1}}\right): \operatorname{Pr}\left[X_{1}^{n} \in \mathcal{S}_{n}\right]=\epsilon-\mu .
$$

Transmitter 1: Assume it observes the sequence $X_{1}^{n}=x_{1}^{n}$. If $x_{1}^{n} \notin \mathcal{S}_{n}$, it looks for indices $m_{1} \geq 1$ satisfying $\left(u_{1}^{n}\left(m_{1}\right), x^{n}\right) \in \mathcal{T}_{\mu}^{n}\left(P_{U_{1} X_{1}}\right)$, randomly picks one of these indices, and sends its corresponding bit-string $\mathrm{M}_{1}=\operatorname{string}\left(m_{1}\right)$ both to Transmitter 2 and the Receiver. Otherwise, it sends the single-bit string $\mathrm{M}_{1}=[0]$.

Transmitter 2: Assume it observes the sequence $X_{2}^{n}=x_{2}^{n}$ and receives the bit-string message $M_{1}=m_{1}$ from Transmitter 1 . If $\mathrm{m}_{1}=[0]$, then it sends the bit-string message $\mathrm{M}_{2}=[0]$. Else, if $m_{1}=\operatorname{dec}\left(\mathrm{m}_{1}\right) \geq 1$, it looks for an index $m_{2} \geq 1$ satisfying $\left(u_{1}^{n}\left(m_{1}\right), u_{2}^{n}\left(m_{2} \mid m_{1}\right), x_{2}^{n}\right) \in \mathcal{T}_{\mu}^{n}\left(P_{U_{1} U_{2} X_{2}}\right)$. It randomly picks one of these indices and sends its corresponding bit-string $\mathrm{M}_{2}=\operatorname{string}\left(m_{2}\right)$ to the Receiver. Otherwise, it sends $\mathrm{M}_{2}=[0]$.

Receiver: Assume it observes the sequence $Y^{n}=y^{n}$ and receives messages $M_{1}=m_{1}$ and $M_{2}=m_{2}$. If any of the bit-strings $m_{1}$ or $m_{2}$ equals [0], it declares $\hat{\mathcal{H}}=1$. Else, it sets $m_{i}=\operatorname{dec}\left(\mathrm{m}_{i}\right)$, for $i=1,2$, and checks if $\left(u_{1}^{n}\left(m_{1}\right), u_{2}^{n}\left(m_{2} \mid m_{1}\right), y^{n}\right) \in \mathcal{T}_{\mu}^{n}\left(P_{U_{1} U_{2} Y}\right)$. It declares $\hat{\mathcal{H}}=0$ if the condition is verified, and $\hat{\mathcal{H}}=1$ otherwise.

\section{A. Analysis}

Notice first that when $X_{1}^{n} \notin \mathcal{S}_{n}$, our variable-length scheme acts like the fixed-length one in [10]. We denote by $\hat{\mathcal{H}}^{\mathrm{ZL}}$ the hypothesis guessed by the scheme in [10].

The type-I error probability of our scheme satisfies

$$
\begin{aligned}
\alpha_{n}= & \operatorname{Pr}[\hat{\mathcal{H}}=1 \mid \mathcal{H}=0] \\
= & \operatorname{Pr}\left[\hat{\mathcal{H}}=1, X_{1}^{n} \in \mathcal{S}_{n} \mid \mathcal{H}=0\right] \\
& +\operatorname{Pr}\left[\hat{\mathcal{H}}=1, X_{1}^{n} \notin \mathcal{S}_{n} \mid \mathcal{H}=0\right] \\
= & \operatorname{Pr}\left[X_{1}^{n} \in \mathcal{S}_{n} \mid \mathcal{H}=0\right] \\
& +\operatorname{Pr}\left[\hat{\mathcal{H}}^{\mathrm{ZL}}=1, X_{1}^{n} \notin \mathcal{S}_{n} \mid \mathcal{H}=0\right] \\
\leq & \epsilon-\mu+\operatorname{Pr}\left[\hat{\mathcal{H}}^{\mathrm{ZL}}=1 \mid \mathcal{H}=0\right] .
\end{aligned}
$$

Since by [10], $\operatorname{Pr}\left[\hat{\mathcal{H}}^{\mathrm{ZL}}=1 \mid \mathcal{H}=0\right] \rightarrow 0$ as $n \rightarrow \infty$, we conclude that for the proposed scheme: $\lim _{n \rightarrow \infty} \alpha_{n} \leq \epsilon$.

The type-II error probability satisfies:

$$
\begin{aligned}
\beta_{n}= & \operatorname{Pr}[\hat{\mathcal{H}}=0 \mid \mathcal{H}=1] \\
= & \operatorname{Pr}\left[\hat{\mathcal{H}}=0, X_{1}^{n} \in \mathcal{S}_{n} \mid \mathcal{H}=1\right] \\
& +\operatorname{Pr}\left[\hat{\mathcal{H}}=0, X_{1}^{n} \notin \mathcal{S}_{n} \mid \mathcal{H}=1\right] \\
= & \operatorname{Pr}\left[\hat{\mathcal{H}}^{\mathrm{ZL}}=0, X_{1}^{n} \notin \mathcal{S}_{n} \mid \mathcal{H}=1\right] \\
\leq & \operatorname{Pr}\left[\hat{\mathcal{H}}^{\mathrm{ZL}}=0 \mid \mathcal{H}=1\right] \\
\leq & 2^{-n\left(I\left(U_{1} U_{2} ; Y\right)+\delta(\mu)\right)},
\end{aligned}
$$

where (31) uses the achievability result in [10] and $\delta(\mu) \rightarrow 0$ as $\mu \rightarrow 0$. Therefore, our scheme achieves the type-II error exponent

$$
\theta \geq I\left(U_{1} U_{2} ; Y\right)+\delta(\mu)
$$

Define $L_{1} \triangleq \operatorname{len}\left(\mathrm{M}_{1}\right)$ and $L_{2} \triangleq \operatorname{len}\left(\mathrm{M}_{2}\right)$. Notice that for sufficiently large blocklengths $n$ and $\mu>0$ :

$$
\begin{aligned}
\mathbb{E}\left[L_{1}\right]= & \mathbb{E}\left[L_{1} \mid X_{1}^{n} \in \mathcal{S}_{n}\right] \operatorname{Pr}\left[X_{1}^{n} \in \mathcal{S}_{n}\right] \\
& +\mathbb{E}\left[L_{1} \mid X_{1}^{n} \notin \mathcal{S}_{n}\right] \operatorname{Pr}\left[X_{1}^{n} \notin \mathcal{S}_{n}\right] \\
\leq & (\epsilon-\mu)+n\left(I\left(U_{1} ; X_{1}\right)+\mu\right) \cdot(1-\epsilon+\mu)
\end{aligned}
$$




$$
\begin{aligned}
& \leq n(1-\epsilon+\mu)\left(I\left(U_{1} ; X_{1}\right)+2 \mu\right) \\
& =n R_{1} .
\end{aligned}
$$

Similarly, for sufficiently large blocklengths $n$ and $\mu>0$ :

$$
\begin{aligned}
\mathbb{E}\left[L_{2}\right]= & \mathbb{E}\left[L_{2} \mid X_{1}^{n} \in \mathcal{S}_{n}\right] \operatorname{Pr}\left[X_{1}^{n} \in \mathcal{S}_{n}\right] \\
& +\mathbb{E}\left[L_{2} \mid X_{1}^{n} \notin \mathcal{S}_{n}\right] \operatorname{Pr}\left[X_{1}^{n} \notin \mathcal{S}_{n}\right] \\
\leq & (\epsilon-\mu)+n\left(I\left(U_{2} ; X_{2} \mid U_{1}\right)+\mu\right) \cdot(1-\epsilon+\mu) \\
= & n\left(I\left(U_{2} ; X_{2} \mid U_{1}\right)+2 \mu\right) \cdot(1-\epsilon+\mu) \\
\leq & n R_{2}
\end{aligned}
$$

Letting $n \rightarrow \infty$ and $\mu \rightarrow 0$ concludes our achievability proof.

\section{Converse Proof to Theorem 1}

Notice first that it suffices to show

$$
\theta_{\epsilon}^{*}\left(R_{1}, R_{2}\right) \leq \max _{\substack{p\left(u_{1} \mid x_{1}\right) p\left(u_{2} \mid u_{1}, x_{2}\right): \\ R_{1} \geq(1-\epsilon) I\left(U_{1} ; X_{1}\right) \\ R_{2} \geq(1-\epsilon) I\left(U_{2} ; X_{2} \mid U_{1}\right) \\ U_{1} \leftrightarrow X_{1} \leftrightarrow\left(X_{2}, Y\right) \\ U_{2} \leftrightarrow\left(X_{2}, U_{1}\right) \leftrightarrow Y}} I\left(U_{1} U_{2} ; Y\right),
$$

i.e., the Markov chain $U_{2} \leftrightarrow\left(U_{1}, X_{2}\right) \leftrightarrow\left(X_{1}, Y\right)$ in Theorem 1 can be replaced by the weaker Markov chain $U_{2} \leftrightarrow\left(U_{1}, X_{2}\right) \leftrightarrow Y$, because the right-hand side of (42) does not depend on the joint pmf of $U_{2}$ and $X_{1}$. More formally, we can prove the equivalence

$$
\begin{aligned}
& \left(I\left(U_{1} U_{2} ; Y\right), I\left(U_{1} ; X_{1}\right), I\left(U_{2} ; X_{2} \mid U_{1}\right)\right) \\
& U_{1} \leftrightarrow X_{1} \leftrightarrow\left(X_{2}, Y\right) \\
& U_{2} \leftrightarrow\left(U_{1}, X_{2}\right) \leftrightarrow\left(Y, X_{1}\right) \\
& =\bigcup_{\substack{U_{1}, U_{2}: \\
U_{1} \leftrightarrow X_{1} \leftrightarrow\left(X_{2}, Y\right) \\
U_{2} \leftrightarrow\left(U_{1}, X_{2}\right) \leftrightarrow Y}}\left(I\left(U_{1} U_{2} ; Y\right), I\left(U_{1} ; X_{1}\right), I\left(U_{2} ; X_{2} \mid U_{1}\right)\right) .
\end{aligned}
$$

Since the two objective functions coincide and the constraints on the left-hand side (LHS) are more stringent, it suffices to show that the right-hand side (RHS) is included in the LHS. To this end, fix $U_{1}, U_{2}$ satisfying the constraints on the LHS, i.e., the Markov chains $U_{1} \leftrightarrow X_{1} \leftrightarrow\left(X_{2}, Y\right)$ and $U_{2} \leftrightarrow$ $\left(U_{1}, X_{2}\right) \leftrightarrow Y$. Then, construct $\tilde{U}_{1}, \tilde{U}_{2}$ so that

$$
\begin{aligned}
P_{\tilde{U}_{1} \mid X_{1} X_{2} Y}\left(u_{1} \mid x_{1}, x_{2}, y\right) & =P_{U_{1} \mid X_{1}}\left(u_{1} \mid x_{1}\right) \\
P_{\tilde{U}_{2} \mid \tilde{U}_{1} X_{1} X_{2} Y}\left(u_{2} \mid u_{1}, x_{1}, x_{2}, y\right) & =P_{U_{2} \mid U_{1} X_{2}}\left(u_{2} \mid u_{1}, x_{2}\right),
\end{aligned}
$$

and thus satisfying the Markov chains on the RHS: $\tilde{U}_{1} \leftrightarrow X_{1} \leftrightarrow\left(X_{2}, Y\right)$ and $\tilde{U}_{2} \leftrightarrow\left(\tilde{U}_{1}, X_{2}\right) \leftrightarrow\left(Y, X_{1}\right)$. The proof is concluded by noting that

$$
\begin{aligned}
I\left(\tilde{U}_{1} ; X_{1}\right) & =I\left(U_{1} ; X_{1}\right), \\
I\left(\tilde{U}_{2} ; X_{2} \mid \tilde{U}_{1}\right) & =I\left(U_{2} ; X_{2} \mid U_{1}\right), \\
I\left(\tilde{U}_{1} \tilde{U}_{2} ; Y\right) & =I\left(U_{1} U_{2} ; Y\right) .
\end{aligned}
$$

Equalities (46) and (47) hold trivially by construction. Equality (48) holds because $P_{\tilde{U}_{1} X_{2}}=P_{U_{1} X_{2}}$ and $P_{\tilde{U}_{2} Y \mid \tilde{U}_{1} X_{2}}=$ $P_{U_{2} \mid U_{1} X_{2}} \cdot P_{Y \mid X_{2}}=P_{U_{2} Y \mid U_{1} X_{2}}$.

We proceed to show that (42) holds. Fix $\theta<\theta_{\epsilon}^{*}\left(R_{1}, R_{2}\right)$, a sequence of encoding and decision functions satisfying the
type-I and type-II error constraints, a blocklength $n$, and a small number $\eta \geq 0$. Define:

$$
\begin{aligned}
\mathcal{B}_{n}(\eta) \triangleq & \left\{\left(x_{1}^{n}, x_{2}^{n}\right):\right. \\
& \left.\quad \operatorname{Pr}\left[\hat{\mathcal{H}}=0 \mid X_{1}^{n}=x_{1}^{n}, X_{2}^{n}=x_{2}^{n}, \mathcal{H}=0\right] \geq \eta\right\}, \\
\mu_{n} \triangleq & n^{-\frac{1}{3}} \\
\mathcal{D}_{n}(\eta) \triangleq & T_{\mu_{n}}^{n}\left(P_{X_{1} X_{2}}\right) \cap \mathcal{B}_{n}(\eta) .
\end{aligned}
$$

By constraint (10) on the type-I error probability:

$$
\begin{aligned}
1-\epsilon \leq & \sum_{x_{1}^{n}, x_{2}^{n}} \operatorname{Pr}\left[\hat{\mathcal{H}}=0 \mid X_{1}^{n}=x_{1}^{n}, X_{2}^{n}=x_{2}^{n}, \mathcal{H}=0\right] \\
\leq & \sum_{\left(x_{1}^{n}, x_{2}^{n}\right) \in \mathcal{B}_{n}(\eta)} P_{X_{1}^{n} X_{2}^{n}}\left(x_{1}^{n}, x_{2}^{n}\left(x_{1}^{n}, x_{2}^{n}\right)\right. \\
& +\sum_{\left(x_{1}^{n}, x_{2}^{n}\right) \notin \mathcal{B}_{n}(\eta)} \operatorname{Pr}\left[\hat{\mathcal{H}}=0 \mid X_{1}^{n}=x_{1}^{n}, X_{2}^{n}=x_{2}^{n}, \mathcal{H}=0\right] \\
\leq & P_{X_{1}^{n} X_{2}^{n}}\left(\mathcal{B}_{n}(\eta)\right)+\eta\left(1-P_{X_{1}^{n} X_{2}^{n}}\left(\mathcal{B}_{n}(\eta)\right)\right) .
\end{aligned}
$$

Thus we have:

$$
P_{X_{1}^{n} X_{2}^{n}}\left(B_{n}(\eta)\right) \geq \frac{1-\epsilon-\eta}{1-\eta} .
$$

Moreover, by [12, Lemma 2.12], the probability that $\left(X_{1}^{n}, X_{2}^{n}\right)$ lie in the jointly strongly typical set $\mathcal{T}_{\mu_{n}}^{(n)}\left(P_{X_{1} X_{2}}\right)$ satisfies

$$
P_{X_{1} X_{2}}^{n}\left(\mathcal{T}_{\mu_{n}}^{(n)}\left(P_{X_{1} X_{2}}\right)\right) \geq 1-\frac{\left|\mathcal{X}_{1}\right|\left|\mathcal{X}_{2}\right|}{2 \mu_{n} n},
$$

and since for any events $A$ and $B$,

$$
\operatorname{Pr}(A \cap B) \geq \operatorname{Pr}(A)+\operatorname{Pr}(B)-1,
$$

then by (51), (55) and (56), we obtain

$$
P_{X_{1}^{n} X_{2}^{n}}\left(D_{n}(\eta)\right) \geq \frac{1-\epsilon-\eta}{1-\eta}-\frac{\left|\mathcal{X}_{1}\right|\left|\mathcal{X}_{2}\right|}{2 \mu_{n} n} \triangleq \Delta_{n} .
$$

We define the random variables $\left(\tilde{\mathrm{M}}_{1}, \tilde{\mathrm{M}}_{2}, \tilde{X}_{1}^{n}, \tilde{X}_{2}^{n}, \tilde{Y}^{n}\right)$ as the restriction of the random variables $\left(\mathrm{M}_{1}, \mathrm{M}_{2}, X_{1}^{n}, X_{2}^{n}, Y^{n}\right)$ to $\left(X_{1}^{n}, X_{2}^{n}\right) \in D_{n}(\eta)$. The probability distribution of the former tuple is given by:

$$
\begin{aligned}
& P_{\tilde{\mathrm{M}}_{1} \tilde{\mathrm{M}}_{2} \tilde{X}_{1}^{n} \tilde{X}_{2}^{n} \tilde{Y}^{n}}\left(\mathrm{~m}_{1}, \mathrm{~m}_{2}, x_{1}^{n}, x_{2}^{n}, y^{n}\right) \triangleq \\
& P_{X_{1}^{n} X_{2}^{n} Y^{n}}\left(x_{1}^{n}, x_{2}^{n}, y^{n}\right) \cdot \frac{\mathbb{1}\left\{x_{1}^{n}, x_{2}^{n} \in D_{n}(\eta)\right\}}{P_{X_{1}^{n} X_{2}^{n}\left(D_{n}(\eta)\right)}} \\
& \cdot \mathbb{1}\left\{\phi_{1}\left(x_{1}^{n}\right)=\mathrm{m}_{1}\right\} \cdot \mathbb{1}\left\{\phi_{2}\left(x_{2}^{n}, \phi_{1}\left(x_{1}^{n}\right)\right)=\mathrm{m}_{2}\right\},
\end{aligned}
$$

leading to the following inequalities:

$$
\begin{gathered}
P_{\tilde{X}_{1}^{n} \tilde{X}_{2}^{n}}\left(x_{1}^{n}, x_{2}^{n}\right) \leq P_{X_{1} X_{2}}^{n}\left(x_{1}^{n}, x_{2}^{n}\right) \Delta_{n}^{-1}, \\
P_{\tilde{\mathrm{M}}_{1} \tilde{\mathrm{M}}_{2}}\left(\mathrm{~m}_{1}, \mathrm{~m}_{2}\right) \leq P_{\mathrm{M}_{1} \mathrm{M}_{2}}\left(\mathrm{~m}_{1}, \mathrm{~m}_{2}\right) \Delta_{n}^{-1}, \\
P_{\tilde{Y}^{n}}\left(y^{n}\right) \leq P_{Y}^{n}\left(y^{n}\right) \Delta_{n}^{-1} \\
D\left(P_{\tilde{X}_{1}^{n} \tilde{X}_{2}^{n}} \| P_{X_{1} X_{2}}^{n}\right) \leq \log \Delta_{n}^{-1} .
\end{gathered}
$$




\section{A. Single-Letter Characterization of Rate Constraints}

Define the following random variables:

$$
\tilde{L}_{i} \triangleq \operatorname{Len}\left(\tilde{\mathrm{M}}_{i}\right), \quad i=1,2 .
$$

By the rate constraints (5) and (6), we have for $i=1,2$ :

$$
\begin{aligned}
n R_{i} & \geq \mathbb{E}\left[L_{i}\right] \\
& \geq \mathbb{E}\left[L_{i} \mid\left(X_{1}^{n}, X_{2}^{n}\right) \in D_{n}(\eta)\right] P_{X_{1}^{n} X_{2}^{n}}\left(D_{n}(\eta)\right) \\
& =\mathbb{E}\left[\tilde{L}_{i}\right] P_{X_{1}^{n} X_{2}^{n}}\left(D_{n}(\eta)\right) \\
& \geq \mathbb{E}\left[\tilde{L}_{i}\right] \Delta_{n},
\end{aligned}
$$

where the last inequality follows by (58). Moreover, by definition, $\tilde{L}_{i}$ is a function of $\tilde{\mathrm{M}}_{i}$, for $i=1,2$, so we can upper bound the entropy of $\tilde{\mathrm{M}}_{i}$ as follows:

$$
\begin{aligned}
H\left(\tilde{\mathrm{M}}_{i}\right) & =H\left(\tilde{\mathrm{M}}_{i}, \tilde{L}_{i}\right) \\
& =H\left(\tilde{\mathrm{M}}_{i} \mid \tilde{L}_{i}\right)+H\left(\tilde{L}_{i}\right) \\
& =\sum_{l_{i}} \operatorname{Pr}\left[\tilde{L}_{i}=l_{i}\right] H\left(\tilde{\mathrm{M}}_{i} \mid \tilde{L}_{i}=l_{i}\right)+H\left(\tilde{L}_{i}\right) \\
& \leq \sum_{l_{i}} \operatorname{Pr}\left[\tilde{L}_{i}=l_{i}\right] l_{i}+H\left(\tilde{L}_{i}\right) \\
& =\mathbb{E}\left[\tilde{L}_{i}\right]+H\left(\tilde{L}_{i}\right) \\
& \leq \frac{n R_{i}}{\Delta_{n}}+H\left(\tilde{L}_{i}\right) \\
& \leq \frac{n R_{i}}{\Delta_{n}}+\frac{n R_{i}}{\Delta_{n}} h_{b}\left(\frac{\Delta_{n}}{n R_{i}}\right) \\
& =\frac{n R_{i}}{\Delta_{n}}\left(1+h_{b}\left(\frac{\Delta_{n}}{n R_{i}}\right)\right),
\end{aligned}
$$

where (74) holds by (68), and (75) holds since the maximum possible entropy of $\tilde{L}_{i}$ is obtained by a geometric distribution of mean $\mathbb{E}\left[\tilde{L}_{i}\right]$, which is further bounded by $\frac{n R_{i}}{\Delta_{n}}[13$, Theorem 12.1.1].

On the other hand, we lower bound the entropy of $\tilde{\mathrm{M}}_{1}$ as:

$$
\begin{aligned}
H\left(\tilde{\mathrm{M}}_{1}\right) \geq & I\left(\tilde{\mathrm{M}}_{1} ; \tilde{X}_{1}^{n} \tilde{X}_{2}^{n}\right)+D\left(P_{\tilde{X}_{1}^{n} \tilde{X}_{2}^{n}} \| P_{X_{1} X_{2}}^{n}\right)+\log \Delta_{n} \\
= & H\left(\tilde{X}_{1}^{n} \tilde{X}_{2}^{n}\right)+D\left(P_{\tilde{X}_{1}^{n} \tilde{X}_{2}^{n}}^{n} \| P_{X_{1} X_{2}}^{n}\right) \\
& -H\left(\tilde{X}_{1}^{n} \tilde{X}_{2}^{n} \mid \tilde{\mathrm{M}}_{1}\right)+\log \Delta_{n} \\
\geq & n\left[H\left(\tilde{X}_{1, T} \tilde{X}_{2, T}\right)+D\left(P_{\tilde{X}_{1, T} \tilde{X}_{2, T}} \| P_{X_{1} X_{2}}\right)\right] \\
& -\sum_{t=1}^{n} H\left(\tilde{X}_{1, t} \tilde{X}_{2, t} \mid \tilde{\mathrm{M}}_{1} \tilde{X}_{1}^{t-1} \tilde{X}_{2}^{t-1}\right)+\log \Delta_{n} \\
= & n\left[H\left(\tilde{X}_{1, T} \tilde{X}_{2, T}\right)+D\left(P_{\tilde{X}_{1, T} \tilde{X}_{2, T}} \| P_{X_{1} X_{2}}\right)\right] \\
& -\sum_{t=1}^{n} H\left(\tilde{X}_{1, t} \tilde{X}_{2, t} \mid \tilde{U}_{1, t}\right)+\log \Delta_{n} \\
= & n\left[H\left(\tilde{X}_{1, T} \tilde{X}_{2, T}\right)+D\left(P_{\tilde{X}_{1, T}} \tilde{X}_{2, T} \| P_{X_{1} X_{2}}\right)\right] \\
& -n H\left(\tilde{X}_{1, T} \tilde{X}_{2, T} \mid \tilde{U}_{1, T}, T\right)+\log \Delta_{n} \\
= & n\left[H\left(\tilde{X}_{1} \tilde{X}_{2}\right)+D\left(P_{\tilde{X}_{1} \tilde{X}_{2}}|| P_{X_{1} X_{2}}\right)\right] \\
& -n H\left(\tilde{X}_{1} \tilde{X}_{2} \mid U_{1}\right)+\log \Delta_{n} \\
= & n\left[I\left(U_{1} ; \tilde{X}_{1} \tilde{X}_{2}\right)+D\left(P_{\tilde{X}_{1} \tilde{X}_{2}}|| P_{X_{1} X_{2}}\right)\right]+\log \Delta_{n}(83
\end{aligned}
$$

$$
\geq n\left[I\left(U_{1} ; \tilde{X}_{1}\right)+\frac{1}{n} \log \Delta_{n}\right] .
$$

Here, (77) holds by (63); (79) holds by the super-additivity property in [14, Proposition 1] and by the chain rule; (80) holds by defining $\tilde{U}_{1 t} \triangleq\left(\tilde{\mathrm{M}}_{1}, \tilde{X}_{1}^{t-1}, \tilde{X}_{2}^{t-1}\right)$; (81) holds by defining $T$ uniform over $\{1, \ldots, n\}$ independent of all other random variables; and (82) holds by defining $U_{1} \triangleq\left(\tilde{U}_{1 T}, T\right)$, $\tilde{X}_{1} \triangleq \tilde{X}_{1, T}$, and $\tilde{X}_{2} \triangleq \tilde{X}_{2, T}$.

Similarly,

$$
\begin{aligned}
H\left(\tilde{\mathrm{M}}_{2}\right) & \geq I\left(\tilde{\mathrm{M}}_{2} ; \tilde{X}_{1}^{n} \tilde{X}_{2}^{n} \mid \tilde{\mathrm{M}}_{1}\right) \\
& =\sum_{t=1}^{n} I\left(\tilde{\mathrm{M}}_{2} ; \tilde{X}_{1, t} \tilde{X}_{2, t} \mid \tilde{\mathrm{M}}_{1} \tilde{X}_{1}^{t-1} \tilde{X}_{2}^{t-1}\right) \\
& =\sum_{t=1}^{n} I\left(\tilde{U}_{2, t} ; \tilde{X}_{1, t} \tilde{X}_{2, t} \mid \tilde{U}_{1, t}\right) \\
& =n I\left(\tilde{U}_{2, T} ; \tilde{X}_{1, T} \tilde{X}_{2, T} \mid \tilde{U}_{1, T} T\right) \\
& =n I\left(\tilde{U}_{2, T} T ; \tilde{X}_{1, T} \tilde{X}_{2, T} \mid \tilde{U}_{1, T} T\right) \\
& =n I\left(U_{2} ; \tilde{X}_{1} \tilde{X}_{2} \mid U_{1}\right) \\
& \geq n I\left(U_{2} ; \tilde{X}_{2} \mid U_{1}\right) .
\end{aligned}
$$

Here, (85) holds since $\tilde{M}_{2}$ is function of $\tilde{X}_{2}^{n}$ and $\tilde{M}_{1} ;(86)$ holds by the chain rule; (87) holds by the definition of $\tilde{U}_{1 t}$, and by defining $\tilde{U}_{2 t} \triangleq \tilde{\mathrm{M}}_{2}$; and (91) holds by defining $U_{2} \triangleq$ $\left(\tilde{U}_{2 T}, T\right)$.

Combining (76) with (84) and (91) yields:

$$
\begin{aligned}
& R_{1} \geq \frac{I\left(U_{1} ; \tilde{X}_{1}\right)+\frac{1}{n} \log \Delta_{n}}{\left(1+h_{b}\left(\frac{\Delta_{n}}{n R_{1}}\right)\right)} \cdot \Delta_{n} \\
& R_{2} \geq \frac{I\left(U_{2} ; \tilde{X}_{2} \mid U_{1}\right)}{\left(1+h_{b}\left(\frac{\Delta_{n}}{n R_{2}}\right)\right)} \cdot \Delta_{n} .
\end{aligned}
$$

\section{B. Upper Bounding the Type-II Error Exponent}

Define for each $\left(m_{1}, m_{2}\right)$ the set

$$
\mathcal{A}_{n}\left(\mathrm{~m}_{1}, \mathrm{~m}_{2}\right) \triangleq\left\{y^{n}:\left(\mathrm{m}_{1}, \mathrm{~m}_{2}, y^{n}\right) \in \mathcal{A}_{n}\right\},
$$

and its Hamming neighborhood:

$$
\begin{array}{r}
\hat{\mathcal{A}}_{n}^{\ell_{n}}\left(\mathrm{~m}_{1}, \mathrm{~m}_{2}\right) \triangleq\left\{\tilde{y}^{n}: \exists y^{n} \in \mathcal{A}_{n}\left(\mathrm{~m}_{1}, \mathrm{~m}_{2}\right)\right. \\
\text { s.t. } \left.d_{H}\left(y^{n}, \tilde{y}^{n}\right) \leq \ell_{n}\right\}
\end{array}
$$

for some real number $\ell_{n}$ satisfying $\lim _{n \rightarrow \infty} \ell_{n} / n=0$ and $\lim _{n \rightarrow \infty} \ell_{n} / \sqrt{n}=\infty$.

Since by definitions (49) and (51), for all $\left(x_{1}^{n}, x_{2}^{n}\right) \in \mathcal{D}_{n}$ :

$$
P_{\tilde{Y}^{n} \mid \tilde{X}_{1}^{n} \tilde{X}_{2}^{n}}\left(\mathcal{A}_{n}\left(\mathrm{~m}_{1}, \mathrm{~m}_{2}\right) \mid x_{1}^{n}, x_{2}^{n}\right) \geq \eta,
$$

then by the blowing-up lemma [15]:

$$
P_{\tilde{Y}^{n} \mid \tilde{X}_{1}^{n} \tilde{X}_{2}^{n}}\left(\hat{\mathcal{A}}_{n}^{\ell_{n}}\left(\mathrm{~m}_{1}, \mathrm{~m}_{2}\right) \mid x_{1}^{n}, x_{2}^{n}\right) \geq 1-\zeta_{n}
$$

for a real number $\zeta_{n}>0$ such that $\lim _{n \rightarrow \infty} \zeta_{n}=0$. Moreover, by taking the expectation over (97):

$$
P_{\tilde{\mathrm{M}}_{1} \tilde{\mathrm{M}}_{2} \tilde{Y}^{n}}\left(\hat{\mathcal{A}}_{n}^{\ell_{n}}\right)=\sum_{\substack{\left(x_{1}^{n}, x_{2}^{n}\right) \in \mathcal{D}_{n} \\\left(\mathrm{~m}_{1}, \mathrm{~m}_{2}\right) \in \mathcal{M}_{1} \times \mathcal{M}_{2}}} P_{\tilde{Y}^{n} \mid \tilde{X}_{1}^{n} \tilde{X}_{2}^{n}}\left(\hat{\mathcal{A}}_{n}^{\ell_{n}}\left(\mathrm{~m}_{1}, \mathrm{~m}_{2}\right) \mid x_{1}^{n}, x_{2}^{n}\right)
$$




$$
\geq 1-\zeta_{n} . \quad \cdot P_{\tilde{X}_{1}^{n} \tilde{X}_{2}^{n} \tilde{\mathrm{M}}_{1} \tilde{\mathrm{M}}_{2}}\left(x_{1}^{n}, x_{2}^{n}, \mathrm{~m}_{1}, \mathrm{~m}_{2}\right)
$$

In addition, using (61) and (62), we have the following:

$$
\begin{aligned}
P_{\tilde{\mathrm{M}}_{1} \tilde{\mathrm{M}}_{2}} & P_{\tilde{Y}^{n}}\left(\hat{\mathcal{A}}_{n}^{\ell_{n}}\right) \\
& \leq P_{\mathrm{M}_{1} \mathrm{M}_{2}} P_{Y}^{n}\left(\hat{\mathcal{A}}_{n}^{\ell_{n}}\right) \cdot \Delta_{n}^{-2} \\
& \leq P_{\mathrm{M}_{1} \mathrm{M}_{2}} P_{Y}^{n}\left(\mathcal{A}_{n}\right) \cdot e^{n h_{b}\left(\ell_{n} / n\right)} \cdot p^{\ell_{n}} \cdot|\mathcal{Y}|^{\ell_{n}} \cdot \Delta_{n}^{-2}(100) \\
& =\beta_{n} \cdot F_{n}^{\ell_{n}} \cdot \Delta_{n}^{-2}
\end{aligned}
$$

where $p \triangleq \min _{y, y^{\prime}: P_{Y}\left(y^{\prime}\right)>0} \frac{P_{Y}(y)}{P_{Y}\left(y^{\prime}\right)}$ and $F_{n}^{\ell_{n}} \triangleq e^{n h_{b}\left(\ell_{n} / n\right)} \cdot p^{\ell_{n}}$.

$|\mathcal{Y}|^{\ell_{n}}$. Here, (100) holds by [12, Proof of Lemma 5.1].

By (101) and standard inequalities (see [9, Lemma 1]), we can upper bound the type-II error exponent as follows:

$-\log \beta_{n}$

$$
\begin{aligned}
\leq & -\log P_{\tilde{\mathrm{M}}_{1} \tilde{\mathrm{M}}_{2}} P_{\tilde{Y}^{n}}\left(\hat{\mathcal{A}}_{n}^{\ell_{n}}\right)+\ell_{n} \log F_{n}-2 \log \Delta_{n} \\
\leq & \frac{1}{1-\zeta_{n}}\left(D\left(P_{\tilde{\mathrm{M}}_{1} \tilde{\mathrm{M}}_{2} \tilde{Y}^{n}} \| P_{\tilde{\mathrm{M}}_{1} \tilde{\mathrm{M}}_{2}} P_{\tilde{Y}^{n}}\right)+1\right) \\
& +\ell_{n} \log F_{n}-2 \log \Delta_{n} \\
= & \frac{1}{1-\zeta_{n}}\left(I\left(\tilde{\mathrm{M}}_{1} \tilde{\mathrm{M}}_{2} ; \tilde{Y}^{n}\right)+1\right)+\ell_{n} \log F_{n}-2 \log \Delta_{n}
\end{aligned}
$$

We further upper-bound the term $I\left(\tilde{\mathrm{M}}_{1} \tilde{\mathrm{M}}_{2} ; \tilde{Y}^{n}\right)$ as follows:

$$
\begin{aligned}
I\left(\tilde{\mathrm{M}}_{1} \tilde{\mathrm{M}}_{2} ; \tilde{Y}^{n}\right) & =\sum_{t=1}^{n} I\left(\tilde{\mathrm{M}}_{1} \tilde{\mathrm{M}}_{2} ; \tilde{Y}_{t} \mid \tilde{Y}^{t-1}\right) \\
& \leq \sum_{t=1}^{n} I\left(\tilde{\mathrm{M}}_{1} \tilde{\mathrm{M}}_{2} \tilde{X}_{1}^{t-1} \tilde{X}_{2}^{t-1} \tilde{Y}^{t-1} ; \tilde{Y}_{t}\right) \\
& =\sum_{t=1}^{n} I\left(\tilde{\mathrm{M}}_{1} \tilde{\mathrm{M}}_{2} \tilde{X}_{1}^{t-1} \tilde{X}_{2}^{t-1} ; \tilde{Y}_{t}\right) \\
& =\sum_{t=1}^{n} I\left(\tilde{U}_{1, t} \tilde{U}_{2, t} ; \tilde{Y}_{t}\right) \\
& =n I\left(\tilde{U}_{1, T} \tilde{U}_{2, T} ; \tilde{Y}_{T} \mid T\right) \\
& \leq n I\left(U_{1} U_{2} ; \tilde{Y}\right)
\end{aligned}
$$

where (107) holds by the Markov chain $\tilde{Y}^{t-1} \leftrightarrow$ $\left(\tilde{\mathrm{M}}_{1} \tilde{\mathrm{M}}_{2}, \tilde{X}_{1}^{t-1} \tilde{X}_{2}^{t-1}\right) \leftrightarrow \tilde{Y}_{t}$; (110) follows by the definitions of $\tilde{U}_{1}$ and $\tilde{U}_{2}$ and defining $\tilde{Y}=\tilde{Y}_{T}$.

\section{Establishing the Desired Markov Chains}

We observe the Markov chain $\tilde{U}_{2, t} \leftrightarrow\left(\tilde{U}_{1, t}, \tilde{X}_{2, t}\right) \leftrightarrow \tilde{Y}_{t}$ for any $t$, and thus $U_{2} \leftrightarrow\left(U_{1}, \tilde{X}_{2}\right) \leftrightarrow \tilde{Y}$. The second desired Markov chain $U_{1} \leftrightarrow \tilde{X}_{1} \leftrightarrow\left(\tilde{X}_{2}, \tilde{Y}\right)$ only holds in the limit as $n \rightarrow \infty$. To see this, notice that $\tilde{\mathrm{M}}_{1} \leftrightarrow \tilde{X}_{1}^{n} \leftrightarrow\left(\tilde{X}_{2}^{n}, \tilde{Y}^{n}\right)$ forms a Markov chain and thus:

$$
\begin{aligned}
0= & I\left(\tilde{\mathrm{M}}_{1} ; \tilde{X}_{2}^{n} \tilde{Y}^{n} \mid \tilde{X}_{1}^{n}\right) \\
\geq & H\left(\tilde{X}_{2}^{n} \tilde{Y}^{n} \mid \tilde{X}_{1}^{n}\right)+D\left(P_{\tilde{X}_{1}^{n}} \tilde{X}_{2}^{n} \tilde{Y}^{n} \| P_{X_{1} X_{2} Y}^{n}\right) \\
& +\log \Delta_{n}-H\left(\tilde{X}_{2}^{n} \tilde{Y}^{n} \mid \tilde{X}_{1}^{n} \tilde{\mathrm{M}}_{1}\right) \\
= & n\left[H\left(\tilde{X}_{2, T} \tilde{Y}_{T} \mid \tilde{X}_{1, T}\right)+D\left(P_{\tilde{X}_{1, T} \tilde{X}_{2, T} \tilde{Y}_{T}}|| P_{X_{1} X_{2} Y}\right)\right] \\
& +\log \Delta_{n}-\sum_{t=1}^{n} H\left(\tilde{X}_{2, t} \tilde{Y}_{t} \mid \tilde{X}_{1}^{n} \tilde{X}_{2}^{t-1} \tilde{Y}^{t-1} \tilde{\mathrm{M}}_{1}\right)
\end{aligned}
$$

$$
\begin{aligned}
& \geq n\left[H\left(\tilde{X}_{2, T} \tilde{Y}_{T} \mid \tilde{X}_{1, T}\right)+D\left(P_{\tilde{X}_{1, T} \tilde{X}_{2, T} \tilde{Y}_{T}}|| P_{X_{1} X_{2} Y}\right)\right] \\
& +\log \Delta_{n}-\sum_{t=1}^{n} H\left(\tilde{X}_{2, t} \tilde{Y}_{t} \mid \tilde{X}_{1}^{t} \tilde{X}_{2}^{t-1} \tilde{\mathrm{M}}_{1}\right) \\
& =n\left[H\left(\tilde{X}_{2, T} \tilde{Y}_{T} \mid \tilde{X}_{1, T}\right)+D\left(P_{\tilde{X}_{1, T} \tilde{X}_{2, T} \tilde{Y}_{T}}|| P_{X_{1} X_{2} Y}\right)\right] \\
& +\log \Delta_{n}-\sum_{t=1}^{n} H\left(\tilde{X}_{2, t} \tilde{Y}_{t} \mid \tilde{X}_{1, t} \tilde{U}_{1, t}\right) \\
& =n\left[H\left(\tilde{X}_{2, T} \tilde{Y}_{T} \mid \tilde{X}_{1, T}\right)+D\left(P_{\tilde{X}_{1, T} \tilde{X}_{2, T} \tilde{Y}_{T}} \| P_{X_{1} X_{2} Y}\right)\right] \\
& +\log \Delta_{n}-n H\left(\tilde{X}_{2, T} \tilde{Y}_{T} \mid \tilde{X}_{1, T} \tilde{U}_{1, T} T\right) \\
& =n\left[I\left(\tilde{X}_{2, T} \tilde{Y}_{T} ; \tilde{U}_{1, T} T \mid \tilde{X}_{1, T}\right)\right. \\
& \left.+D\left(P_{\tilde{X}_{1, T} \tilde{X}_{2, T} \tilde{Y}_{T}} \| P_{X_{1} X_{2} Y}\right)\right]+\log \Delta_{n} \\
& =n\left[I\left(\tilde{X}_{2} \tilde{Y} ; U_{1} \mid \tilde{X}_{1}\right)+D\left(P_{\tilde{X}_{1} \tilde{X}_{2} \tilde{Y}} \| P_{X_{1} X_{2} Y}\right)\right]+\log \Delta_{n} \\
& \geq n I\left(\tilde{X}_{2} \tilde{Y} ; U_{1} \mid \tilde{X}_{1}\right)+\log \Delta_{n},
\end{aligned}
$$

where (112) follows by (63) and $P_{\tilde{Y}^{n} \mid \tilde{X}_{1}^{n} \tilde{X}_{2}^{n}}=P_{Y \mid X_{1} X_{2}}^{n}$; (113) holds by the super-additivity property in [14, Proposition 1] and the chain rule; (114) holds since knowledge reduces entropy; and finally (115) and (118) hold by the definitions of $\tilde{U}_{1, t}, \tilde{X}_{2}, \tilde{Y}$, and $U_{1}$. Moreover, since $\frac{1}{n} \log \Delta_{n} \rightarrow 0$ as $n \rightarrow \infty$, then $I\left(\tilde{X}_{2} \tilde{Y} ; U_{1} \mid \tilde{X}_{1}\right) \rightarrow 0$ as $n \rightarrow \infty$.

D. The Limits $n \rightarrow \infty$ and $\eta \rightarrow 0$

To sum up, we have proved so far in (92), (93), (104), (110), and (119) that for all $n \geq 1$ there exists a joint pmf $P_{\tilde{X}_{1} \tilde{X}_{2} \tilde{Y} U_{1} U_{2}}^{(n)}$ (abbreviated as $P^{(n)}$ ) and functions $g_{1}(n)$, $g_{3}(n)$, and $g_{4}(n)$ tending to 0 as $n \rightarrow \infty$ and $g_{2}(n, \eta)$ tending to $(1-\epsilon)$ as $n \rightarrow \infty$ and $\eta \rightarrow 0$, so that

$$
\begin{aligned}
P_{\tilde{X}_{1} \tilde{X}_{2} \tilde{Y} U_{1} U_{2}}^{(n)} & =P_{\tilde{X}_{1} \tilde{X}_{2} \tilde{Y}}^{(n)} \cdot P_{U_{1} \mid \tilde{X}_{1} \tilde{X}_{2}}^{(n)} \cdot P_{U_{2} \mid U_{1} \tilde{X}_{2}}^{(n)}, \\
R_{1} & \geq\left(I_{P^{(n)}}\left(U_{1} ; \tilde{X}_{1}\right)+g_{1}(n)\right) \cdot g_{2}(n, \eta), \\
R_{2} & \geq I_{P^{(n)}}\left(U_{2} ; \tilde{X}_{2} \mid U_{1}\right) \cdot g_{2}(n, \eta), \\
\theta & \leq I_{P^{(n)}}\left(U_{1} U_{2} ; \tilde{Y}\right)+g_{3}(n), \\
I_{P^{(n)}}\left(\tilde{X}_{2} \tilde{Y} ; U_{1} \mid \tilde{X}_{1}\right) & \leq g_{4}(n),
\end{aligned}
$$

where $I_{P^{(n)}}$ indicates that the mutual information should be calculated according to the pmf $P^{(n)}$.

Applying Carathéodory's theorem [11, Appendix C], one can restrict the auxiliary random variables $U_{1}$ and $U_{2}$ to alphabets of sizes

$$
\begin{aligned}
& \left|\mathcal{U}_{1}\right| \leq\left|\mathcal{X}_{1}\right| \cdot\left|\mathcal{X}_{2}\right|+3 \\
& \left|\mathcal{U}_{2}\right| \leq\left|\mathcal{U}_{1}\right| \cdot\left|\mathcal{X}_{2}\right|+1
\end{aligned}
$$

The proof is then concluded by invoking the BolzanoWeierstrass theorem, and by considering a subsequence $P_{\tilde{X}_{1} \tilde{X}_{2} \tilde{Y} U_{1} U_{2}}^{\left(n_{k}\right)}$ that converges to a limiting pmf $P_{X_{1} X_{2} Y U_{1} U_{2}}^{*}$. In fact, by (120) this limiting pmf factorizes as $P_{X_{1} X_{2} Y U_{1} U_{2}}^{*}=$ $P_{X_{1} X_{2} Y}^{*} \cdot P_{U_{1} \mid X_{1}}^{*} \cdot P_{U_{2} \mid U_{1} X_{2}}^{*}$ and satisfies the desired rateconstraints, and moreover $P_{X_{1} X_{2} Y}^{*}=P_{X_{1} X_{2}} \cdot P_{Y \mid X_{1} X_{2}}$ because for any $n \geq 1, P_{\tilde{Y} \mid \tilde{X}_{1} \tilde{X}_{2}}^{(n)}=P_{Y \mid X_{1} X_{2}}$ and $\mid P_{\tilde{X}_{1} \tilde{X}_{2}}-$ $P_{X_{1} X_{2}} \mid \leq \mu_{n}\left(\right.$ since $\left.\left(\tilde{X}_{1}^{n}, \tilde{X}_{2}^{n}\right) \in \mathcal{T}_{\mu_{n}}^{(n)}\left(P_{X_{1} X_{2}}\right)\right)$ with $\mu_{n} \rightarrow 0$ as $n \rightarrow \infty$. 


\section{ACKNOWLEDGMENT}

M. Wigger and M. Hamad acknowledge funding support from the ERC under grant agreement 715111.

\section{REFERENCES}

[1] R. Ahlswede and I. Csiszár, "Hypothesis testing with communication constraints," IEEE Trans. Inf. Theory, vol. 32, pp. 533-542, Jul. 1986.

[2] T. S. Han, "Hypothesis testing with multiterminal data compression," IEEE Trans. Inf. Theory, vol. 33, pp. 759-772, Nov. 1987.

[3] H. Shimokawa, T. Han, and S. I. Amari, "Error bound for hypothesis testing with data compression," in Proc. ISIT, p. 114, Jul. 1994.

[4] M. S. Rahman and A. B. Wagner, "On the optimality of binning for distributed hypothesis testing," IEEE Trans. Inf. Theory, vol. 58, pp. 6282-6303, Oct. 2012.

[5] Y. Xiang and Y. H. Kim, "Interactive hypothesis testing against independence," in Proc. ISIT, pp. 2840-2844, Jun. 2013.

[6] P. Escamilla, M. Wigger, and A. Zaidi, "Distributed hypothesis testing with concurrent detection," in Proc. ISIT, Jun. 2018.

[7] S. Salehkalaibar and M. Wigger, "Distributed hypothesis testing based on unequal-error protection codes," IEEE Trans. Inf. Theory, vol. 66, pp. 4150-41820, Jul. 2020.

[8] S. Salehkalaibar, M. Wigger, and L. Wang, "Hypothesis testing over the two-hop relay network," IEEE Trans. Inf. Theory, vol. 65, pp. 44114433, Jul. 2019.

[9] S. Salehkalaibar and M. Wigger, "Distributed hypothesis testing with variable-length coding," arXiv preprint arXiv:2005.08610, 2020.

[10] W. Zhao and L. Lai, "Distributed testing with cascaded encoders," IEEE Trans. Inf. Theory, vol. 64, no. 11, pp. 7339-7348, 2018.

[11] A. El Gamal and Y. H. Kim, Network Information Theory. Cambridge University Press, 2011.

[12] I. Csiszár and J. Körner, Information theory: coding theorems for discrete memoryless systems. Cambridge University Press, 2011.

[13] T. M. Cover and J. A. Thomas, Elements of Information Theory, 2nd Ed. Wiley, 2006.

[14] H. Tyagi and S. Watanabe, "Strong converse using change of measure arguments," IEEE Trans. Inf. Theory, vol. 66, no. 2, pp. 689-703, 2019.

[15] K. Marton, "A simple proof of the blowing-up lemma," IEEE Trans. Inf. Theory, vol. 32, pp. 445-446, May 1986. 